$\begin{array}{cc}\text { Programa de Pós-Graduação em Engenharia de Produção - PPGEP } & \\ \text { Laboratório de Qualidade de Vida - LaQVida } & \\ \text { Universidade Tecnológica Federal do Paraná - UTFPR } & \text { REVISTA BRASILEIRA DE } \\ \text { Ponta Grossa - PR - Brasil } & \text { QUALIDADE DE VIDA }\end{array}$

\title{
Comparativo da qualidade de vida de acadêmicos de Educação Física de Campinas-SP e Ponta Grossa-PR
}

\section{Comparative of quality of life between Physical Education college students of Campinas-SP and Ponta Grossa-PR}

\author{
Fabrício Cieslak \\ Universidade Federal do Paraná - UFPR - Curitiba - Brasil \\ facieslak@gmail.com \\ Antônio José Grande \\ Universidade Metodista de Piracicaba - UNIMEP - Piracicaba - Brasil \\ grandeto@gmail.com \\ Gustavo Levandoski \\ Universidade Federal do Paraná - UFPR - Curitiba - Brasil \\ glevandoski@gmail.com \\ Alex Pinheiro Gordia \\ Universidade Federal do Recôncavo da Bahia - UFRB - Amargosa - Brasil \\ alexgordia@gmail.com \\ Teresa Maria Bianchini de Quadros \\ Universidade Federal do Recôncavo da Bahia - UFRB - Amargosa - Brasil \\ tetemb@gmail.com \\ Guanis de Barros Vilela Junior \\ Universidade Metodista de Piracicaba - UNIMEP - Piracicaba - Brasil \\ Faculdades Metropolitanas de Campinas - METROCAMP - Campinas - Brasil \\ guanis@gmail.com \\ Neiva Leite \\ Universidade Federal do Paraná - UFPR - Curitiba - Brasil \\ neivaleite@gmail.com
}

\begin{abstract}
Resumo
O objetivo do presente trabalho é analisar a qualidade de vida (QV) de acadêmicos de Educação Física de ambos os sexos e de diferentes estados brasileiros. Participaram do estudo 85 estudantes de Ponta Grossa-PR (46 do sexo masculino e 39 do sexo feminino) e 61 estudantes de Campinas-SP (31 do sexo masculino e 30 do sexo feminino). A QV foi mensurada pelo WHOQOL-bref, para análise dos resultados foi utilizada a sintaxe australiana e a consistência interna através do coeficiente Alfa de Cronbach. Para localizar as diferenças foi utilizado o teste de Kruskal-Wallis, seguido do teste de Mann-Whitney com o valor de p corrigido pela técnica de Bonferroni. A QV foi satisfatória e com os menores valores para o domínio ambiental, foram encontradas diferenças significativas entre os grupos para todos os domínios. Pode-se inferir a necessidade do investimento em políticas públicas.
\end{abstract}

Palavras-chave: acadêmicos, educação física, qualidade de vida, WHOQOL-bref. 


\begin{abstract}
This paper objectifies to analyze the quality of life (QoL) of Physical Education college students of both sexes and the different Brazilian states. Part of the study 85 students of Ponta Grossa-PR (46 males and 39 women) and 61 students of Campinas-SP (31 males and 39 women). The QoL was measured by WHOQOL-bref, for analysis of the results was used Australian syntax and the internal consistency by the Cronbach's alpha. To find the differences were used the Kruskal-Wallis test, followed by the Mann-Whitney test with a value of $p$ corrected by technique of Bonferroni. The QoL was satisfactory and lower values for the environmental, significant differences were found between groups in all areas. We can infer need for investment in public politics.
\end{abstract}

Keywords: college students, physical education, quality of life, WHOQOL-bref.

\title{
1. Introdução
}

A Qualidade de Vida (QV) de uma população é dependente de diversos fatores referentes às suas condições de existência, acesso a certos bens e serviços econômicos e sociais: emprego e renda, educação básica, alimentação adequada, acesso a bons serviços de saúde, saneamento básico, habitação, transporte, entre outros aspectos. Vale ressaltar, que o conceito de bem-estar, de QV, é variável para cada sociedade e decorre conforme a sua cultura (ADRIANO et al., 2000).

Nesse sentido, a Organização Mundial da Saúde (OMS) a QV pode ser conceituada como: "a percepção do indivíduo de sua posição na vida no contexto da cultura e sistema de valores nos quais ele vive e em relação aos seus objetivos, expectativas, padrões e preocupações" (THE WHOQOL GROUP, 1994 apud FLECK et al., 2000, p.179).

Como método avaliativo de QV, a OMS formulou o instrumento World Health Organization Quality of Life - WHOQOL (THE WHOQOL GROUP, 1998), e que tem sido empregado de forma ampla em estudos nacionais (CIESLAK et al., 2007; GORDIA et al., 2007) e internacionais (SAXENA et al., 2001; KAO et al., 2005) como método de mensuração desse componente. Este protocolo apresenta alta aceitação sendo testado e validado em diversos países, inclusive no Brasil (FLECK et al., 2000).

Nesse contexto, o presente estudo objetivou analisar a QV de acadêmicos de Educação Física de ambos os sexos e de dois diferentes estados brasileiros.

\section{Procedimentos metodológicos}

O estudo envolveu um delineamento observacional, descritivo e transversal, sendo realizado nos municípios de Campinas (SP) e Ponta Grossa (PR), no ano de 2006. Participaram do estudo 61 indivíduos (31 do sexo masculino e 30 do sexo feminino) de uma faculdade de Educação Física privada do município de Campinas (SP) e 85 indivíduos (46 do sexo masculino e 39 do sexo feminino) de um curso de Educação Física pública do município de Ponta Grossa (PR). Após detalhado esclarecimento sobre os propósitos dessa investigação, procedimentos utilizados, benefícios e possíveis riscos atrelados, os participantes assinaram termo de consentimento, condicionando sua participação de modo voluntário.

A mensuração da QV dos estudantes foi realizada através da utilização do instrumento WHOQOL, protocolo desenvolvido pelo grupo de estudos de QV da Organização Mundial da Saúde em 1995. Elaborou-se o instrumento denominado WHOQOL-100 (composto de 100 questões e seis domínios) e com validação efetuada em diversos países, entretanto, na busca de um instrumento menos complexo e de fácil aplicabilidade, desenvolveu-se o WHOQOL-bref, um instrumento com a mesma essência do WHOQOL-100, mas com 26 questões circunscritas a quatro domínios.

O referido protocolo constitui-se de 26 questões relativas aos últimos quinze dias anteriores à avaliação (THE WHOQOL GROUP, 1998). Duas questões são referentes à percepção individual 
da qualidade de vida e as demais estão subdivididas em quatro domínios, representando cada uma das 24 facetas que compõem o instrumento original (WHOQOL-100). Os domínios do WHOQOLbref são representados no quadro 1:

Quadro 1 - Domínios e facetas do WHOQOL-bref

\begin{tabular}{|c|c|c|c|}
\hline $\begin{array}{l}\text { Domínio I } \\
\text { Físico }\end{array}$ & $\begin{array}{l}\text { Domínio II } \\
\text { Psicológico }\end{array}$ & $\begin{array}{c}\text { Domínio III } \\
\text { Relações } \\
\text { Sociais } \\
\end{array}$ & $\begin{array}{c}\text { Domínio IV } \\
\text { Meio-Ambiente }\end{array}$ \\
\hline $\begin{array}{c}\text { - Dor e desconforto; } \\
\text { - Energia e fadiga; } \\
\text { - Sono e repouso; } \\
\text { - Mobilidade; } \\
\text { - Atividades da vida } \\
\text { cotidiana; } \\
\text { - Dependência de medicação } \\
\text { e tratamentos; } \\
\text { - Capacidade para o trabalho; }\end{array}$ & $\begin{array}{c}\text { - Sentimentos positivos; } \\
\text { - Pensar; } \\
\text { - Aprender; } \\
\text { - Memória e concentração; } \\
\text { - Auto-estima; } \\
\text { - Imagem corporal e } \\
\text { aparência; } \\
\text { - Sentimentos negativos; } \\
\text { - Espiritualidade, religião e } \\
\text { crenças pessoais; }\end{array}$ & $\begin{array}{c}\text { - Relações } \\
\text { pessoais; } \\
\text { - Suporte } \\
\text { (apoio) social; } \\
\text { - Atividade } \\
\text { sexual; }\end{array}$ & $\begin{array}{c}\text { - Segurança física e proteção; } \\
\text { - Ambiente no lar; } \\
\text { - Recursos financeiros; } \\
\text { - Cuidados de saúde e sociais; } \\
\text { - Oportunidade de adquirir novas } \\
\text { informações e habilidades; } \\
\text { - Participação em/e oportunidades } \\
\text { de recreação/lazer; } \\
\text { - Ambiente físico: poluição, ruído, } \\
\text { trânsito, clima e transporte; }\end{array}$ \\
\hline
\end{tabular}

Fonte: The WHOQOL Group (1998)

Para a análise dos resultados referentes à investigação da QV, adotaram-se os critérios propostos pela equipe australiana do WHOQOL (THE AUSTRALIAN CENTRE FOR POSTTRAUMATIC MENTAL HEALTH, 2003). Foram utilizadas medidas de tendência central e variabilidade para verificar o nível de QV dos indivíduos e o teste de Shapiro-Wilk para a normalidade dos dados, em seguida empregou-se o teste de Kruskal-Wallis com o intuito de localizar as diferenças entre os grupos, para localizar as diferenças específicas utilizou-se o teste de Mann-Whitney com o valor de p corrigido pela técnica de Bonferroni, o nível adotado foi de significância de $p<0,05$. A verificação da consistência interna das respostas do instrumento foi obtida por intermédio do coeficiente de fidedignidade Alfa de Cronbach $(\alpha)$ (STREINER, 2003). O tratamento estatístico foi realizado mediante a utilização do software Statistical Package for the Social Sciences (SPSS, versão 13.0) for Windows.

\section{Resultados e discussão}

Os resultados obtidos através da aplicação do instrumento WHOQOL-bref são apresentados na tabela 1, subdivididos em domínios da seguinte forma: Domínio I - Físico, Domínio II Psicológico, Domínio III - Relações Sociais, Domínio IV - Meio-Ambiente e QV geral:

Tabela 1 - Valores do WHOQOL-bref apresentados por domínios

\begin{tabular}{ccccc}
\hline $\begin{array}{c}\text { Qualidade } \\
\text { de Vida }\end{array}$ & $\begin{array}{c}\text { Feminino } \\
\text { Ponta Grossa }\end{array}$ & $\begin{array}{c}\text { Masculino } \\
\text { Ponta Grossa }\end{array}$ & $\begin{array}{c}\text { Feminino } \\
\text { Campinas }\end{array}$ & $\begin{array}{c}\text { Masculino } \\
\text { Campinas }\end{array}$ \\
\hline $\begin{array}{c}\text { Domínio I } \\
\text { Físico }\end{array}$ & $72,2 \pm 12,5^{\mathrm{b}, \mathrm{d}}$ & $77,2 \pm 10,6^{\mathrm{a}, \mathrm{d}}$ & $75,3 \pm 14,7^{\mathrm{d}}$ & $83,1 \pm 11,0^{\mathrm{a}, \mathrm{b}, \mathrm{c}}$ \\
$\begin{array}{c}\text { Domínio II } \\
\text { Psicológico }\end{array}$ & $68,4 \pm 14,8^{\mathrm{d}}$ & $72,0 \pm 12,2^{\mathrm{d}}$ & $70,5 \pm 15,2^{\mathrm{d}}$ & $78,2 \pm 14,6^{\mathrm{a}, \mathrm{b}, \mathrm{c}}$ \\
$\begin{array}{c}\text { Domínio III } \\
\text { Relações Sociais } \\
\text { Domínio IV }\end{array}$ & $79,7 \pm 16,1^{\mathrm{b}, \mathrm{c}}$ & $73,9 \pm 16,9^{\mathrm{a}, \mathrm{d}}$ & $70,0 \pm 14,1^{\mathrm{a}, \mathrm{d}}$ & $81,9 \pm 16,8^{\mathrm{b}, \mathrm{c}}$ \\
$\begin{array}{c}\text { Meio-Ambiente } \\
\text { QV }\end{array}$ & $63,5 \pm 12,5^{\mathrm{c}}$ & $61,0 \pm 13,1$ & $57,3 \pm 13,4^{\mathrm{a}, \mathrm{d}}$ & $66,7 \pm 14,7^{\mathrm{c}}$ \\
Geral & $70,9 \pm 13,8^{\mathrm{d}}$ & $71,0 \pm 13,1^{\mathrm{d}}$ & $68,3 \pm 14,2^{\mathrm{d}}$ & $77,5 \pm 14,1^{\mathrm{a}, \mathrm{b}, \mathrm{c}}$ \\
\hline
\end{tabular}

Fonte: Autoria própria (2010)

\footnotetext{
${ }^{\text {a }}$ Diferença significante do grupo masculino de Ponta Grossa.

${ }^{\mathrm{b}}$ Diferença significante do grupo feminino de Ponta Grossa.

${ }^{\mathrm{c}}$ Diferença significante do grupo masculino de Campinas.

${ }^{\mathrm{d}}$ Diferença significante do grupo feminino de Campinas.
} 
Os resultados demonstram uma QV geral satisfatória dos participantes, sendo que, o grupo masculino de Campinas $(\chi=77,5+14,1)$ apresentou os melhores valores e com diferenças estatísticas em relação ao grupo feminino de Ponta Grossa $(p=0,029)$, masculino de Ponta Grossa $(p=0,034)$ e feminino de Campinas $(\mathrm{p}=0,0012)$.

Dentre três dos grupos estudados, sobretudo os acadêmicos do sexo masculino de Campinas $(\chi=83,1+11,0)$, foram demonstrados os maiores valores para o Domínio I - Físico e foram encontradas diferenças significativas entre os estudantes do sexo feminino e masculino de Ponta Grossa ( $\mathrm{p}=0,041)$, do sexo feminino de Ponta Grossa e feminino de Campinas ( $\mathrm{p}=0,0001$ ), do sexo masculino de Ponta Grossa e Campinas $(\mathrm{p}=0,015)$ e do sexo masculino e feminino de Campinas $(\mathrm{p}=0,031)$. Esses valores corroboram os dados encontrados no estudo de Nunes e Freire (2006) que avaliaram de modo transversal a QV de 237 cirurgiões-dentistas da Secretaria Municipal de Saúde de Goiânia (GO) e indicaram as maiores médias para o presente domínio.

O Domínio II - Psicológico apresentou os maiores valores para o grupo do sexo masculino de Campinas $(\chi=78,2+14,6)$ e que diferiu significativamente do grupo feminino de Ponta Grossa $(\mathrm{p}=0,005)$, do masculino de Ponta Grossa $(\mathrm{p}=0,024)$ e do feminino de Campinas $(\mathrm{p}=0,040)$. No estudo de Bittencourt et al. (2004), na avaliação da QV de pacientes renais foram apresentados valores semelhantes para o domínio psicológico.

Para o Domínio III - Relações Sociais os grupos que indicaram os maiores valores foram o masculino de Campinas $(\chi=81,9+16,8)$ com diferenças significativas para o masculino de Ponta Grossa $(\mathrm{p}=0,028)$ e feminino de Campinas $(\mathrm{p}=0,002)$, e o grupo feminino de Ponta Grossa $(\chi=79,7+16,1)$ com diferenças significativas entre os estudantes do sexo masculino de Ponta Grossa $(\mathrm{p}=0,027)$ e os do sexo feminino de Campinas $(\mathrm{p}=0,008)$. Na pesquisa de Saupe et al. (2004) foram avaliados 825 acadêmicos de enfermagem e foram encontrados os maiores valores para o presente domínio. Da mesma forma, Penteado e Pereira (2007), na avaliação da QV e saúde vocal de 128 professores do ensino público de Rio Claro encontraram os maiores valores para o Domínio III Relações Sociais.

Os piores valores apontados neste estudo estiveram presentes em todos os grupos para o Domínio IV - Meio-Ambiente, com diferenças significantes entre o grupo feminino de Campinas e de Ponta Grossa ( $\mathrm{p}=0,036)$ e o masculino e feminino de Campinas $(\mathrm{p}=0,012)$. No estudo de Gordia, Quadros e Vilela Junior (2006), na análise da QV de 65 indivíduos do sexo masculino pertencentes ao exército brasileiro, os menores valores foram para o presente domínio. Similarmente, Wang et al. (2006) avaliaram a QV de 13.083 adultos do Taiwan e foram observadas as piores médias para o domínio ambiental em ambos os sexos e sendo o principal ponto vulnerável da amostra estudada.

\section{Considerações finais}

O presente estudo possibilitou uma visão reflexiva na análise da QV no âmbito de acadêmicos do curso de Educação Física. Por intermédio dos resultados obtidos, pode-se inferir que os estudantes do sexo masculino de Campinas apresentaram os maiores valores na QV geral.

Para todos os grupos foram relatados os menores valores no domínio ambiental. Esses resultados atestam equivalência com outras análises e evidenciam a necessidade do investimento em políticas públicas, considerando que as sociedades atuais possuem graves problemas estruturais e relacionados a fatores econômicos, educacionais e da saúde.

\section{Referências}

ADRIANO, J. R. et al. A construção de cidades saudáveis: uma estratégia viável para a melhoria da qualidade de vida? Ciência \& Saúde Coletiva, Rio de Janeiro, v.5, n.1, p.53-62, 2000. 
BITTENCOURT, Z. Z. L. C. et al. Qualidade de vida em transplantados renais: importância do enxerto funcionante. Revista de Saúde Pública, São Paulo, v.38, n.5, p.732-734, out. 2004.

CIESLAK, F. et al. Estudo da qualidade de vida de mulheres idosas participantes do programa da universidade aberta à terceira idade na cidade de Ponta Grossa-PR. Lecturas, Educácion Física y Deportes, Buenos Aires, v.12, n.10, oct. 2007.

GORDIA, A. P.; QUADROS, T. M. B. Q.; VILELA JUNIOR, G. B. Quality of life and physical fitness of individuals in the Brazilian army. The FIEP Bulletin, Foz do Iguaçu, v.76, n.1, p.78-80, jan. 2006.

GORDIA, A. P. et al. Comparação da qualidade de vida de mulheres idosas praticantes e não praticantes de exercício físico. Lecturas, Educácion Física y Deportes, Buenos Aires, v.11, n.3, mar. 2007.

FLECK, M. P. A. et al. Aplicação da versão em português do instrumento abreviado de avaliação da qualidade de vida "WHOQOL-bref". Revista de Saúde Pública, São Paulo, v.34, n.2, p.178183, abr. 2000.

KAO, S. et al. WHOQOL-bref as predictors of mortality: a two-year follow-up study at veteran homes. Quality of Life Research, Los Angeles, v.14, n.6, p.1443-1454, aug. 2005.

NUNES, M. F.; FREIRE, M. C. M. Qualidade de vida de cirurgiões-dentistas que atuam em um serviço público. Revista de Saúde Pública, São Paulo, v.40, n.6, p.1019-1026, dez. 2006.

PENTEADO, R. Z.; PEREIRA, I. M. T. B. Qualidade de vida e saúde vocal de professores. Revista de Saúde Pública, São Paulo, v.41, n.2, p.236-243, abr. 2006.

SAUPE, R. et al. Qualidade de vida dos acadêmicos de enfermagem. Revista Latino-Americana de Enfermagem, Ribeirão Preto, v.12, n.4, p.636-642, jul./ago. 2004.

SAXENA, S. et al. The WHO quality of life assessment instrument (WHOQOL-bref): the importance of its items for cross-cultural research. Quality of Life Research, Los Angeles, v.10, n.8, p.711-721, sep. 2001.

STREINER, D.L. Starting the beginning: an introduction to coefficient alpha and internal consistency. Journal of Personality Assessment, Mahwah, v.80, n.1, p.99-103, feb. 2003.

THE AUSTRALIAN CENTRE FOR POSTRAUMATIC MENTAL HEALTH. Trauma related research, training and policy development. The University of Melbourne. 2003. Disponível em: <http://www.acpmh.unimelb.edu.au/whoqol/whoqol-bref_5.html>. Acesso em: 09 jul. 2006.

THE WHOQOL GROUP. Development of the World Health Organization WHOQOL-bref. Quality of Life Assessment. Psychological Medicine, Leicester, v.28, n.3, p.551-558, may 1998.

WANG, W. C. et al. Validating, improving reliability, and estimating correlation of the four subscales in the WHOQOL-bref using multidimensional Rasch analysis. Quality of Life Research, Los Angeles, v.15, n.4, p.607-620, may 2006. 